\title{
Constructive methods for the design and labeling of four-dimensional modulations
}

\author{
Fernando Alves Rodrigues, Member, IEEE, Guilherme Temporão and Jean Pierre von der Weid, Member, IEEE,
}

\begin{abstract}
A constructive method employing the Hopf fibration formalism in the design of high order modulations has recently been reported. In this paper, we demonstrate through examples that not only the design but also the labeling of these constellations can be parameterized using discrete Hopf fibres. The angular sampling of such discrete Hopf fibres has been found to provide a common geometric variety that can be shared by both the signal and the coding space of these modulations.
\end{abstract}

Index Terms-Four-Dimensional Modulation, Coherent fiber optic communication, Discrete Hopf fibration, Labeling, Covering code.

\section{INTRODUCTION}

$\mathbf{I}$ $\mathrm{N}$ the recent scenario of coherent optical communications the design of uncoded modulations has been performed mainly through the permutation of vector coordinates [1], [2]. The simplicity and efficiency explains why this method has gained great prominence in optical communication systems. Notwithstanding, with the advent of polarization multiplexing [3], [4], the boost in the application of high-order modulations has presented new challenges in fiber optic communications. From a historical perspective, several approaches have been made into this topic [5], [6], [7], but despite these efforts complexity is still evident when one needs to account for integrated requirements in the optical and electrical domains. To address this challenge, a constructive method for high-order modulations using the Hopf fibration between spheres was recently reported [8], [9], [10]. Now, we go further into this topic presenting some extensions of these constructive methods in the design and labeling of 4D constellations. From [11] we can verify that the design of high-order modulations can be optimized through the rotation and translation of constellation symbols defined by polytopes. Following a resembling geometric approach, the proposed methods demonstrated here are based mainly on two mathematical tools. The first is the formalism of the

Fernando A. Rodrigues is with the Center for Telecommunication Studies (CETUC), Pontifical Catholic University of Rio de Janeiro (PUC-Rio) as a Ph.D. student and the National Institute of Metrology, Quality an Technology (Inmetro) as a Technologist Researcher.

email:farodrigues@cetuc.puc-rio.br

Guilherme P. Temporão is with the Department of Electrical Engineering (DEE) and the Center for Telecommunication Studies (CETUC), Pontifical Catholic University of Rio de Janeiro (PUC-Rio).

email:temporao@opto.cetuc.puc-rio.br

Jean Pierre von der Weid is with the Department of Electrical Engineering (DEE) and the Center for Telecommunication Studies (CETUC), Pontifical Catholic University of Rio de Janeiro (PUC-Rio).

email:vdweid@cetuc.puc-rio.br

Digital Object Identifier: 10.14209/jcis.2018.26
Hopf Fibration [12], [13], [14], [15] developed by Heinz Hopf in 1931. Roughly, this method can be considered as the decomposition of a geometric space in subspaces called Hopf fibres. The second tool is the embedding vertex concept applied to regular polytopes [16]. In Section (II) and (II-B) basic properties of the Hopf formalism are demonstrated in the context of classical coherent optical communications [17]. The present work shows that the Hopf fibration is not only suitable for the description of modulations but also it can be used in the labeling of $4 \mathrm{D}$ symbols. Both the design and labeling process account for integrated requirements in the optical (polarization) and electrical (amplitude and phase) domains. In order to demonstrate this premise we recall in section (IV) an example of application where both the Hopf fibration and the embedded vertex methods were used to expand the 6PolSK-QPSK modulation to a 14PolSK-8PSK arrangement. In section (V), we remember how the Cayley-Dickson construction of quaternions can be used for the partitioning of 4D constellations into QAM representations. It is also demonstrated that from these QAM representations we can extract qualitative metrics and shape properties of 4D modulations. Differently from previous works, here we present a simple method for the design of base 2 constellations. In section (VI), is presented how the symmetry of points in the fibration base space can influence in the requirements of the DAC. Although our focus in this work has been the design of 4D modulations, both the Hopf fibration and the embedded vertex methods can be extended to $8 \mathrm{D}$ and even for $16 \mathrm{D}$ modulations [18].

\section{THE HOPF FIBRATION FORMALISM}

From the field of topology in mathematics, there are four widely known Hopf fibrations between spheres [12], [19]:

$$
\begin{aligned}
& S^{0} \rightarrow S^{1} \stackrel{\pi}{\rightarrow} S^{1} ; \\
& S^{1} \rightarrow S^{3} \stackrel{\pi}{\rightarrow} S^{2} ; \\
& S^{3} \rightarrow S^{7} \stackrel{\pi}{\rightarrow} S^{4} ; \\
& S^{7} \rightarrow S^{15} \stackrel{\pi}{\rightarrow} S^{8}
\end{aligned}
$$

where the referred notation means (for the first case) that the space $S^{1}$ is bundled by great circles $S^{0}$ with a base space $S^{1}$, in the second case the space $S^{3}$ is bundled by great circles $S^{1}$ with a base space $S^{2}$. For the other cases the same relations apply [12], [15]. In the present work, targeting the construction of four-dimensional modulations, the focus will be given to the discrete fibration $S^{1} \rightarrow S^{3} \stackrel{\pi}{\rightarrow} S^{2}$. In this context, if the sphere $S^{3}$ is defined as follows:

$$
S^{3}=\left\{z=\left(z_{1}, z_{2}\right) \in \mathbb{C} \times \mathbb{C}:\left|z_{1}\right|^{2}+\left|z_{2}\right|^{2}=1\right\}
$$


the Hopf map can be formulated in complex coordinates as:

$$
h: \mathbb{C} \times \mathbb{C} \rightarrow \mathbb{C} \times \mathbb{R}, h\left(z_{1}, z_{2}\right)=\left(2 z_{1} z_{2}, z_{1} z_{1}-z_{2} z_{2}\right)
$$

where $z_{1}$ and $z_{2}$ are two complex numbers of the form:

$$
z_{1}=\left(x_{1}+i x_{2}\right) ; \quad z_{2}=\left(x_{3}+i x_{4}\right)
$$

doing so, the Hopf map in real coordinates can be written as:

$$
\begin{gathered}
H: R^{4} \rightarrow R^{4} \\
H\left(x_{1}, x_{2}, x_{3}, x_{4}\right)=\left(s_{0}, s_{1}, s_{2}, s_{3}\right)
\end{gathered}
$$

The unit sphere $S^{3} \subset R^{4}$ described by Equation $x_{1}^{2}+x_{2}^{2}+x_{3}^{2}+$ $x_{4}^{2}=r^{2}$ is the locus of four-dimensional vectors, where $x_{i}$ are Cartesian coordinates, and $r$ is the radius of the hypersphere.

The notation of Equation (5) was borrowed from [17] in order to represent a physical meaning in the context of classical coherent optical communications. As noted before, providing the condition $x_{1}^{2}+x_{2}^{2}+x_{3}^{2}+x_{4}^{2}=1$ with $S^{2} \subset R^{3}$, the Poincaré sphere can be used as the base space $S^{2}$ of a Hopf fibration $S^{1} \rightarrow S^{3} \stackrel{\pi}{\rightarrow} S^{2}$.

$$
\begin{aligned}
& s_{0}=x_{1}^{2}+x_{2}^{2}+x_{3}^{2}+x_{4}^{2}=1 ; \\
& s_{1}=x_{1}^{2}+x_{2}^{2}-x_{3}^{2}-x_{4}^{2} ; \\
& s_{2}=2\left(x_{1} x_{3}+x_{2} x_{4}\right) ; \\
& s_{3}=2\left(x_{2} x_{3}-x_{1} x_{4}\right)
\end{aligned}
$$

From the perspective of pure mathematics, the Equation (6) is the well known direct Hopf map. Furthermore, since the coordinates $\left(x_{1}, x_{2}, x_{3}, x_{4}\right)$ can represent real quantities in the classical phase space of the optical field, this equation provides an explicit map between the optical and electrical domains of coherent 4D modulations, with $\left(s_{0}, s_{1}, s_{2}, s_{3}\right)$ representing real measurable quantities of the light polarization.

\section{A. The inverse Hopf map}

To explore the capabilities of the Hopf map in the construction of optical constellations we need to formalize the inverse Hopf map $S^{2} \rightarrow S^{3}$. For our purpose, it is also convenient to follow the notation used in [15], describing the $S^{3}$ space in toroidal coordinates with three independent angles $\phi, \psi$ and $\theta$.

$$
\begin{aligned}
& x_{1}=r \sin (\theta) \cos (\phi) ; \\
& x_{2}=r \sin (\theta) \sin (\phi) ; \\
& x_{3}=r \cos (\theta) \cos (\psi) ; \\
& x_{4}=r \cos (\theta) \sin (\psi) ;
\end{aligned}
$$

em que $\theta \in[0, \pi / 2], \phi \in[0,2 \pi]$ e $\psi \in[0,2 \pi]$.

As denoted in [20], [15], for fixed values of $\theta$, the Equation (7) describes the surface of a torus. So, if a linear relation between the two parameters $\phi$ and $\psi$ holds then there will be only one independent value, which will parameterize a curve in the $S^{3}$ torus. Besides that, if the relation between $\phi$ and $\psi$ result in constant values, then $\psi$ will define a set of great circles that never intersect. In this case, if we choose $\theta$ to be the angle of latitude of a $S^{2}$ sphere, the $S^{3}$ counterpart will be fibred by parallel great circles. The fibration we have just described is illustrated in Figure (1), where a stereographic projection was used to visualize the pre-image of the Hopf fibres [14]. Basis points were defined over two crossed circles conforming 126 points in $S^{2}$.

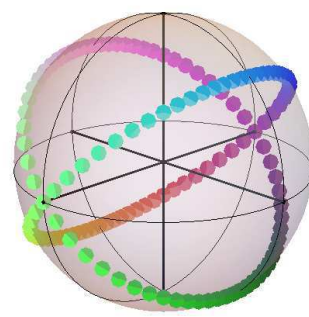

(a)

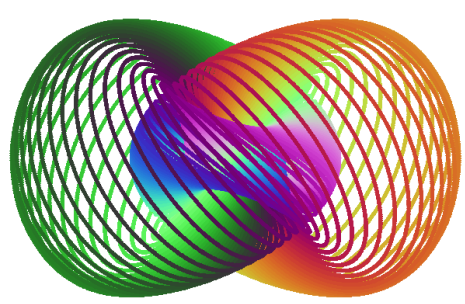

(b)
Fig. 1. (a) 126 points (Hopf fibres) defined over two crossed circles in the sphere $S^{2}$ (b) Stereographic projection of the resulting Hopf fibration (Hopf torus) on the sphere $S^{3}$.

Considering the toroidal coordinates with $r=1$, the Hopf map restricted to $S^{3}$ can be written as:

$$
H(\theta, \phi, \psi)=(\sin (a) \cos (b), \sin (a) \sin (b),-\cos (a))
$$

were $a=2 \theta$ and $b=\phi-\psi$.

In order to harmonize the notation, we recall from [21] that a general element of the $S U(2)$ group can be concisely represented by means of an exponential function employing Pauli spin matrices.

$$
U_{2}=\exp \left(\frac{\mathbf{i} \sigma_{3} \phi}{2}\right) \exp \left(\frac{\mathbf{i} \sigma_{1} \theta}{2}\right) \exp \left(\frac{\mathbf{i} \sigma_{2} \phi}{2}\right)
$$

being Pauli spin matrices in the form:

$$
\sigma_{1}=\left[\begin{array}{cc}
1 & 0 \\
0 & -1
\end{array}\right], \quad \sigma_{2}=\left[\begin{array}{cc}
0 & 1 \\
1 & 0
\end{array}\right], \quad \sigma_{3}=\left[\begin{array}{cc}
0 & -i \\
i & 0
\end{array}\right]
$$

As demonstrated in the Appendix (A), the exponential map parameterized by Euler angles relates $\mathrm{SU}(2)$ with $\mathrm{SO}(3)$ (the group of rotations in $R^{3}$ ). Moreover, still according to [21], the resulting equation for $U^{2}$ can be interpreted as arising from the Hopf map $S^{3} \rightarrow S^{2}$ :

$$
U 2=\left(\begin{array}{cc}
\cos \left(\frac{\theta}{2}\right) e^{i\left(\frac{\phi+\psi}{2}\right)} & i \sin \left(\frac{\theta}{2}\right) e^{i\left(\frac{\phi-\psi}{2}\right)} \\
-i \sin \left(\frac{\theta}{2}\right) e^{-i\left(\frac{\phi-\psi}{2}\right)} & \cos \left(\frac{\theta}{2}\right) e^{-i\left(\frac{\phi+\psi}{2}\right)}
\end{array}\right)
$$

where $(\theta, \phi)$ and $\psi$ are the Euler angles.

Concerning that the $S U(2)$ group corresponding to a rotation is isomorphic with the group of unit quaternions, we can associate the Equation (11) with the general form:

$$
U_{2}=\left(\begin{array}{cc}
z_{1} & z_{2} \\
-\bar{z}_{2} & \bar{z}_{1}
\end{array}\right), \quad z_{1}, z_{2} \in \mathbb{C}, \quad\left|z_{1}\right|^{2}+\left|z_{2}\right|^{2}=1
$$


were quantities $z_{1}, z_{2}$ are the trigonometric equations:

$$
\begin{aligned}
& z_{1}=\cos \left(\frac{\theta}{2}\right) \exp \left(i\left(\frac{\psi+\phi}{2}\right)\right) \\
& z_{2}=\sin \left(\frac{\theta}{2}\right) \exp \left(i\left(\frac{\psi-\phi}{2}\right)\right)
\end{aligned}
$$

Now, remembering that Equation (11) is extensively used in polarization optics, the pair $(\theta, \phi)$ can be identified with the polar angles in the Poincaré sphere. Using relations introduced in Equation (13), we can verify that the angular quantities $(\theta, \phi, \psi)$ in $S^{3}$ obeys a common metric with its counterpart in $S^{2}$. This angular metric is defined as follows:

$$
\begin{aligned}
\theta=\frac{\pi}{2}-\frac{\widetilde{\theta}}{2} ; & 0 \leq \widetilde{\theta} \leq \pi ; \\
\phi=\widetilde{\psi}+\frac{\widetilde{\phi}}{2} ; & 0 \leq \widetilde{\psi}+\frac{\widetilde{\phi}}{2}<2 \pi ; \\
\psi=\widetilde{\psi}-\frac{\widetilde{\phi}}{2} ; & 0 \leq \widetilde{\psi}-\frac{\widetilde{\phi}}{2}<2 \pi ;
\end{aligned}
$$

The angular parameters $(\widetilde{\theta}, \widetilde{\phi})$ in (14) can be applied to the second part of the equality in Equation (8).

$$
H(\theta, \phi, \psi)=(\sin (\widetilde{\theta}) \cos (\widetilde{\phi}), \sin (\widetilde{\theta}) \sin (\widetilde{\phi}), \cos (\widetilde{\theta}))
$$

Finally, recalling Equation (4) and expanding terms of the Equation (13), the inverse Hopf map can be realized by means of the following parametric equations:

$$
\begin{aligned}
& x_{1}=\cos \left(\frac{\theta}{2}\right) \cos \left(\frac{\psi+\phi}{2}\right) ; \\
& x_{2}=\cos \left(\frac{\theta}{2}\right) \sin \left(\frac{\psi+\phi}{2}\right) ; \\
& x_{3}=\sin \left(\frac{\theta}{2}\right) \cos \left(\frac{\psi-\phi}{2}\right) ; \\
& x_{4}=\sin \left(\frac{\theta}{2}\right) \sin \left(\frac{\psi-\phi}{2}\right) ;
\end{aligned}
$$

From now on, we will denote Equation (33) as the inverse Hopf map $\operatorname{IHM}(\theta, \phi, \psi)$, where $\left(x_{1}, x_{2}, x_{3}, x_{4}\right)$ represents the coordinates of an arbitrary 4D vector and the angle $\psi$ defines, for fixed values of $(\widetilde{\theta}, \widetilde{\phi})$, the coordinates of a great circle that is the locus of the respective Hopf fibre $S^{1}$. As already noted, the angle $\widetilde{\theta}$ defines a common metric between the spheres $S^{2}$ and $S^{3}$. This is done in such a way that the distance between two points in the base space equals by half the distance between two planes where the associated Hopf fibres reside [15]. Conversely, two great circles separated by an angular distance $\alpha$ in the sphere $S^{3}$ are mapped through the Hopf fibration to two points separated by a geodesic arc $2 \alpha$ in the sphere $S^{2}$ [22]. From the $\mathrm{SO}(3) \leftrightarrow \mathrm{SU}(2)$ groups perspective this metric holds with the double cover property.
B. The Hopf fibration as an engineering tool in classical optical communications

The ability to fill a geometric space with defined subspaces is related to the problem of sphere packings [23], [20]. In the specific case of the fibration $S^{1} \rightarrow S^{3} \stackrel{\pi}{\rightarrow} S^{2}$, the Hopf fibres are spheres of dimension 1 that fill the geometric space of the hypersphere of dimension 3. This packing of hyperspheres establishes a close relation between Hopf fibres and information theory. To reinforce this concept, some references that contextualize Hopf fibres, light polarization [19] and 4D modulations will be presented in the next sections. Using the $\operatorname{IHM}(\theta, \phi, \psi)$ formulation we can identify the coordinates of all $4 \mathrm{D}$ coplanar vectors that reside in a specific great circle. This procedure is equivalent, therefore, to sample 4D vectors from discrete Hopf circles. The mapping depends, however, on the parameters convention. Using the parametrization of Equation (33), we have constructed $\mathbf{n P o l S K}-\mathbf{m P S K}$ modulations by defining a group of $\mathbf{n}$ points in the $S^{2}$ sphere in conjunction with a group of $\mathbf{m}$ discrete values of the angle $\psi$. The process has started when Stokes vectors over the Poincaré sphere were used in the definition of basis points in $S^{2}$ space. The results presented in the Section (IV) provides evidence that through this mapping, we can perform operations equivalent to rotations and scaling of modulation arrangements. Notwithstanding, to use the Hopf formalism from an engineering perspective we need to harmonize the notation used until here with that commonly used in physics and fiber optic communication.

The Stokes parameters $\left(s_{1}, s_{2}, s_{3}\right)$, will be associated to the spherical coordinates in the form:

$$
\begin{aligned}
& s_{1}=r \sin (\widetilde{\theta}) \cos (\widetilde{\phi}) ; \\
& s_{2}=r \sin (\widetilde{\theta}) \sin (\widetilde{\phi}) ; \quad \widetilde{\theta}=\left[-\frac{\pi}{2}, \frac{\pi}{2}\right] ; \widetilde{\phi}=[0, \pi] ; \\
& s_{3}=r \cos (\widetilde{\theta}) ;
\end{aligned}
$$

where $r$ denotes the radius of the sphere and the pair $(\widetilde{\theta}, \widetilde{\phi})$ represents, respectively, the angles of latitude and longitude in the unit sphere. As we have already pointed out, since the Stokes parameters are physically measurable quantities, using them as coordinates of the basis points of a Hopf fibration provides a physical meaning to a mathematical entity.

So, given the Stokes parameters in the form of Equation (17), one can extract the angles $(\widetilde{\theta}, \widetilde{\phi})$ using the following relations:

$$
\widetilde{\theta}=\frac{1}{2}\left[\operatorname{sgn}\left(s_{1}\right) \arccos \left(\frac{s_{3}}{r}\right)\right], \quad \widetilde{\phi}=\arctan \left(\frac{s_{2}}{s_{1}}\right)
$$

where $\operatorname{sgn}\left(s_{1}\right)$ indicates the signal of the parameter $s_{1}$, for $s_{1} \neq 0$. Due to singularities in the points of latitude $(0,0,1)$ and $(0,0,-1)$ we need to apply a proper rule for $\psi$.

$$
\widetilde{\psi} \rightarrow \widetilde{\psi}-\frac{\pi}{4} ; \quad \text { for }[\widetilde{\theta}=0, \widetilde{\phi}= \pm \pi]
$$


Established the mathematical conventions for the different vector representations in the $S^{2}$ base, we can introduce the Hopf formalism in the mapping of points from the $S^{2}$ sphere to circles in the $S^{3}$ space. The $\operatorname{IHM}(\theta, \phi, \psi)$ will thus relate the state of polarized light to the classical (geometric) phase space where the 4D vectors reside [17]. This procedure allows the construction of optical modulations with polarization states defined a priori. In the next section, we will demonstrate through simple algebraic manipulations that Equation (6) can also be related to the simplified transfer function of PMQ-MZM (Polarization Multiplexed Quadrature Mach-Zehnder) modulators [24], [25], [26].

\section{HOPF MAP AND THE TRANSFER FUNCTION OF PMQ-MZM MODULATORS}

The success of PMQ-MZM components stems from its ability to encode, in the classical sense, a complete description of the electromagnetic light field. From the formulae in [24], the relation between the PMQ-MZM input electric field $E_{i n}$ and the respective output $E_{\text {out }}$, can be described by the following transfer function:

$$
\begin{gathered}
\frac{E_{\text {out }}}{E_{\text {in }}}=\frac{1}{2 \sqrt{2}}\left\{\left(X_{1}+i X_{2}\right)+\left[\left(X_{3}+i X_{4}\right) \cdot e^{j \theta}\right]\right\} \\
X_{n}=\cos \left[\left(\frac{x_{n}+\text { Vbias }}{2 V_{\pi}}\right) \pi\right], n=(1,2,3,4)
\end{gathered}
$$

where quantities $X_{n}$ are representing the transfer function of the four nested Mach-Zehnder on the PMQ-MZM structure. The cosine function of $X_{n}$ are indicating that these Mach-Zehnders are working as amplitude modulators (i.e., in the "push-pull" mode). $x_{n}$ are input drive voltages (quaternion coordinates in Equation (33)), $V_{\text {bias }}$ is the DC bias voltage and $V_{\pi}$ is the reference driving voltage that induces a phase shift of $\pi$ in each Mach-Zehnder. The polarization rotator is represented by the quantity $e^{j \theta}$. Additionally, for real valuated outputs of $X_{n}$ in (20) we can expect that the orthogonality between $X_{1}$ and $X_{2}$ will be maintained. The same applying to $X_{3}$ and $X_{4}$.

We recall now the parametrization of the Hopf map using the formulation described by [14], [27]. According to [27], in the Cayley-Dickson form, unit quaternions $\left(x_{1}, x_{2}, x_{3}, x_{4}\right)$ are represented by two complex numbers $z_{1}$ and $z_{2}$, as denoted by Equation (4). Following this notation, quaternions can represent two orthogonal cartesian planes. The angular relation between the complex numbers $z_{1}$ and $z_{2}$ needs to guarantee that the Hopf map will reside on a unit sphere [14], that is the condition $\left|z_{1}\right|^{2}+\left|z_{2}\right|^{2}=1$ and $r_{1}^{2}+r_{2}^{2}=1$. This is the same condition used in Equation (4), so again a common solution for $z_{1}$ and $z_{2}$ is by using trigonometric functions in order that $r_{1}=\cos (\theta / 2)$ and $r_{2}=\sin (\theta / 2)$. Consequently, we can write the Equation (13) in the form:

$$
\left[\cos \left(\frac{\theta}{2}\right) \exp (i \xi 1), \sin \left(\frac{\theta}{2}\right) \exp (i \xi 2)\right] ;\left\{\begin{array}{l}
0 \leqslant \theta \leqslant \pi \\
\xi 1, \xi 2 \in \operatorname{Re}
\end{array}\right.
$$

For fixed values of $\theta$, Equation (21) is the cartesian product of two circles and in most cases this formulation describes a torus. Finally, observing equations (20) and (21) we find that the Hopf map is close related to the simplified transfer function of a PMQ-MZM denoted in (20). In this way, angles $(\xi 1, \xi 2)$ on equation (21) may be identified with the (IQ) quadratures of the modulator. In turn, the angle $\theta$ in (21) may be identified with the polarization rotator in (20). So, remarking the bias setup in the push-pull mode and the unit vector condition for the electrical input signal, these relations explains from our analysis how the Hopf map $S^{3} \rightarrow S^{2}$ can trace out the polarization state of signals modulated by a simplified model of PMQ-MZM devices. Those relations demonstrated here gives a physical meaning to Equation (6) in the context of coherent optical communications. In the next section an example of application of the formalism presented here will be used in the construction of a 14PolSK-8PSK modulation. As will be demonstrated, the 14PolSK-8PSK brings together the 6PolSK-8PSK and 8PolSK-8PSK symbols in the same modulation arrangement. A remarkable feature that will be evidenced is the fact that the 6PolSK-8PSK arrangement is actually the realization of two congruent 6PolSK-QPSK arrays [22]. This means that the accumulation of the 6PolSK-QPSK symbols with a rigid rotation of itself (vertices of the 24-Cell polytope) generates the 6PolSK-8PSK constellation.

\section{14POLSK-8PSK USING DISCRETE HOPF FIBRATION}

A possible way to construct rate-adaptive modulations is on defining an arrangement of symbols as the accumulation (embedding) of vertex polytopes [16]. An important example is the 6PolSK-QPSK modulation. The geometry of this modulation is strongly associated with the 24-Cell polytope and this polytope can, in turn, be considered as the union of two other polytopes: the 4D hypercube (tesseract) and the 16-Cell polytope. When Equation (6) is used to perform the Hopf map of the 24 symbols of a 6PolSK-QPSK modulation, the related quaternions will project the following six points in the sphere $S^{2}$ :

$$
\begin{aligned}
s \in S^{2}=[ & (0,1,0),(0,0,1),(0,0,-1), \\
& (0,-1,0),(1,0,0),(-1,0,0)]
\end{aligned}
$$

where the set of points $s$ are geometrically arranged as an octahedron in the unit sphere. Alternatively, these points defines the position of $3 \mathrm{D}$ vectors representing classical states of the polarized light (Stokes vectors).

Now, in order to expand the 6PolSK-QPSK arrangement, we simply concatenate new vertices to the illustrated octahedron and after that, given the resulting set of vectors, we perform the inverse Hopf map. To accomplish this task in a structured way, vertices of the polytope Tetrakis Hexahedron [28] were considered as a reference.

The Tetrakis Hexahedron, in its standard form, consists of the union of an octahedron with a hexahedron (cube). So, the two disjoint polytopes embedded in its 14 vertices are inscribed in a three-dimensional unit sphere as shown in Figure (2). From another point of view, if we take the six vertices from 


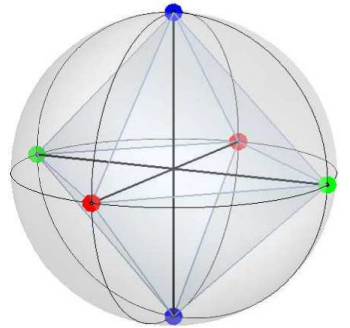

(a)

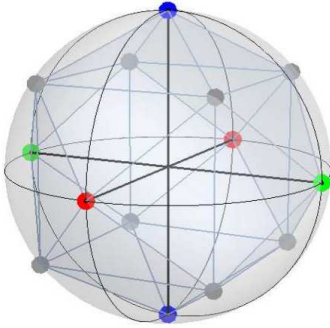

(b)
Fig. 2. Graphical representation of (a) vertices of the 6PolSK-QPSK modulation and (b) vertices of the 14PolSK symbols in the $S^{2}$ sphere after the accumulation of 08 vertices (hexahedron).

the 6PolSK-QPSK projection and properly concatenate eight vertices of a regular hexahedron, we will obtain the Tetrakis Hexahedron as shown in Figure (2b). The inverse Hopf map of the Tetrakis Hexahedron will project the arrangement of symbols for the 14PolSK-mPSK modulation, where $m$ denotes the number of samples of the angle $\psi$ in Equation (33). The two way mapping that we just described was performed using Equations (6), (33), (17) and (18). The discrete Hopf fibres were sampled in 08 equally spaced discrete values of $\psi$. The illustration of the 14PolSK-8PSK symbols in $S^{2}$ and the stereographic projection of the respective fibration can be visualized in Figure (3). The result of the sampled discrete Hopf Fibration is illustrated in Figure (3b).

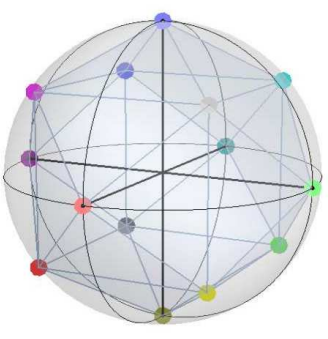

(a)

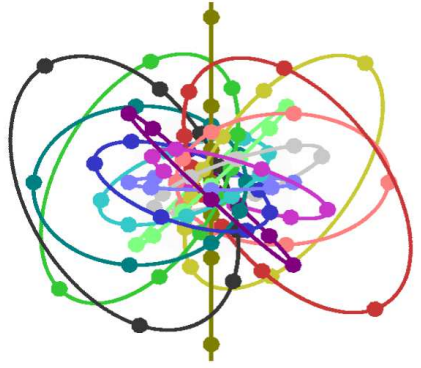

(b)
Fig. 3. (a) The Tetrakis Hexahedron constellation in the $S^{2}$ sphere and (b) the inverse Hopf map (IHM) using the Tetrakis Hexahedron basis points with 8 samples each.

Equation (33) was sampled for the following values of $\psi$ :

$$
\begin{aligned}
& \psi_{\text {sample }_{1}}=\left\{0, \frac{\pi}{2}, \pi, \frac{3 \pi}{2}\right\} \\
& \psi_{\text {sample }_{2}}=\left\{\frac{\pi}{4}, \frac{3 \pi}{4}, \frac{5 \pi}{4}, \frac{7 \pi}{4}\right\}
\end{aligned}
$$

In the space spanned by the discrete fibration, each set $\psi_{\text {sample }}$ showed in (23) generates symbols of a QPSK (4PSK) array [22], joining these sets led to a 8PSK arrangement. On the other hand, from the perspective of the base space, the 14PolSK-8PSK is the union of 6PolSK-8PSK and 8PolSK-8PSK (see [29] for the QPSK version). The distance between the great circles shown in Figure (3b) are defined by the distance between the related basis points shown in
Figure (3a), obeying the metric described in the Section (II-A). As anticipated in Section (II-B), if only the 6PolSK-8PSK symbols are considered and we alternately select those vectors obtained from the sets $\psi_{\text {sample }_{1}}$ and $\psi_{\text {sample }_{2}}$, two congruent 6PolSK-QPSK arrays are obtained, rotated by the angle $\pi / 4$. So, based on the parametrization that we have used, this reasoning can be extended to any set of PSK symbols obtained from the constructive method presented here.

\section{QAM PARTITIONS AND THE CAYLEY-DICKSON REPRESENTATION FOR QUATERNIONS}

If we consider the 112 symbols of the 14PolSK-8PSK as a single constellation, each quaternion obtained from Equation (33) can be represented as two complex numbers just as in the Equation (4). This property of the Cayley-Dickson representation for quaternions [27], [30] can therefore be used to map 4D symbols into two-dimensional partitions (QAM). As an example, consider for instance the graphical representation of the entire 14PolSK-8PSK constellation shown in Figure (4).
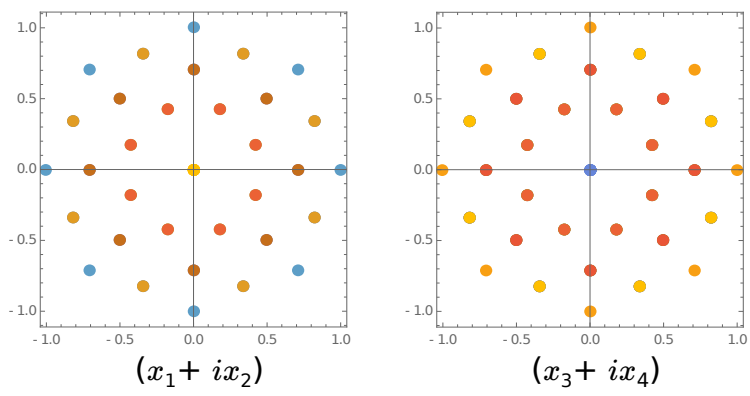

Fig. 4. QAM partition of the 14PolSK-8PSK obtained from the Cayley-Dickson representation of the unit quaternions.

Using the static performance metrics presented in Table (I) [31], where $M$ is the number of constellation points, $N$ is the dimension of the modulation, $d$ is the minimum (Euclidean) distance between symbols, $E_{b}$ is the energy per bit and $E$ is the average energy per symbol. We have found that for the

TABLE I

PERFORMANCE METRIC FOR CONSTELLATIONS

\begin{tabular}{|c|c|c|}
\hline Spectral Efficiency & Power Efficiency & Figure of Merit \\
\hline $\mathrm{SE}=\frac{\log _{2}(M)}{N / 2} \mathrm{bit} / \mathrm{s} / \mathrm{Hz}$ & $\mathrm{PE}=\frac{d^{2}}{4 E_{b}}$ & $\mathrm{CFM}=\frac{d^{2} \mathrm{~N}}{2 \mathrm{E}}$ \\
\hline
\end{tabular}

14PolSK-8PSK, the spectral efficiency (SE) is 3.4, the power efficiency (PE) is 0.4 and the constellation figure of merit (CFM) is 0.478 .

Notwithstanding, these results can be seen in a different way when the 14PolSK-8PSK symbols are considered as an adaptive arrangement of disjoint constellations. In the Figure (5), we have illustrated some constellations symbols of known modulations that are incorporated (embedded) in the 14PolSK-8PSK arrangement: The diagram in Figure (5) also illustrates the rotated versions of the 6PolSK-QPSK and 


$$
14 \text { PolSK-8PSK } \rightarrow\left\{\begin{array}{l}
\text { 6PolSK-8PSK }\left\{\begin{array}{l}
\text { 6PolSK-QPSK }\left\{\begin{array}{l}
\text { PDM-QPSK } \\
\text { PS-QPSK }
\end{array}\right. \\
6 \text { PolSK-QPSK }
\end{array}\right. \\
\text { 8PolSK-8PSK }\left\{\begin{array}{l}
8 \text { PolSK-QPSK } \\
8 \text { PolSK-QPSK }
\end{array}\right.
\end{array}\right.
$$

Fig. 5. Hierarchical diagram of known constellations embedded in the arrangement 14PolSK-8PSK, organized according to the number of symbols.

8PolSK-QPSK constellations. It is important to note that the PS-QPSK constellation embedded in the 6PolSK-QPSK array is considered the most energy efficient $4 \mathrm{D}$ modulation [32], while the PDM-QPSK is the modulation used in the well-known 100G [3] standard. Some properties of the 8PolSK-QPSK was reported by [29]. As noted by [6], the difference between PolSK and PDM transmission schemes is defined based on how the polarization partitions are transmitted. If transmitted alternately we will have a PolSK scheme, if transmitted simultaneously we will have a PDM scheme. It is, therefore, a concluding remark that the 14PolSK-8PSK constellation can be used as a hierarchical adaptive modulation. This arrangement may be composed, for example, of the following modulations: PS-QPSK, PDM-QPSK, 6PolSK-QPSK, 6PolSK-8PSK, 8PolSK-QPSK, 8PolSK-8PSK and 14PolSK-8PSK itself.

\section{A. Using the Hopf map in the construction of base 2 constellations}

So far, we have extracted relevant properties from the 14PolSK-8PSK geometry. Despite that good properties, the problem here is that this constellation with its 112 symbols is not base 2. Fortunately, another advantage of the discrete Hopf fibration method is that we can make a different number of samples in specific Hopf fibres. This property, related to a factorization process, will be explored further in a numerical example targeting the construction of a constellation with 128 symbols. For illustrative purposes, we will use the same tetrakis hexahedron basis due to the fact that it has a non base 2 number of points. In this sense, what we are looking for is a sum of factors $n_{1}, m 1, n_{2}, m_{2}$ in the general form:

$$
\left(n_{1} \text { PolSK- } m_{1} \text { PSK }\right)+\left(n_{2} \text { PolSK- } m_{2} \text { PSK }\right)
$$

In the specific case, of 128 symbols we are interested in:

$$
\left(n_{1} \cdot m_{1}\right)+\left(n_{2} \cdot m_{2}\right)=128
$$

Therefore, the condition $n_{1}+n_{2}=14$, imply an immediate solution with $n_{1}=12, n_{2}=2, m_{1}=8$ and $m_{2}=16$ :

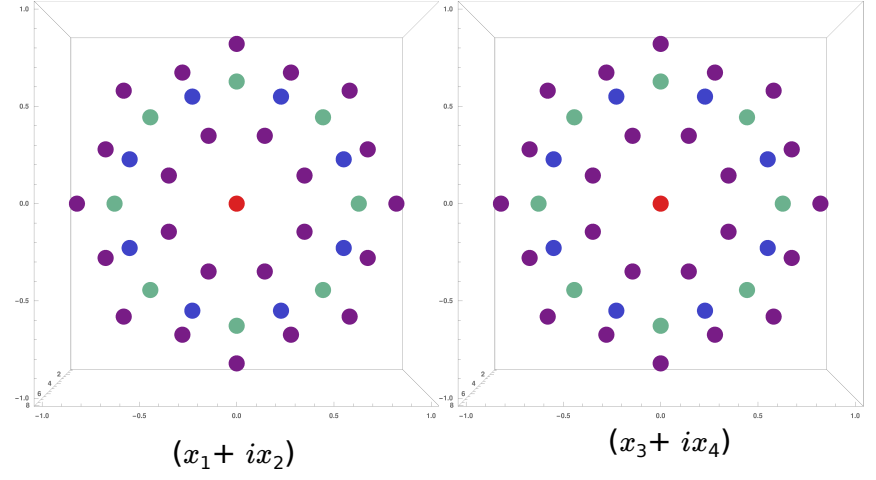

Fig. 6. QAM partition of the array (12PolSK-8PSK) + (2PolSK-16PSK) obtained from the Cayley-Dickson representation of the unit quaternions.

In summary, 12 vertices of the tetrakis hexahedron will be projected in the 4D space with 8 angular samples and the remaining 2 vertices will be projected with 16 angular samples. The resulting constellation has now 128 symbols, retaining the property of unitary norm. The Figure (6) shows the QAM partitions of the constellation obtained by the factorization method. Using the static performance metrics from Table (I), the $\mathrm{SE}$ is 3.5 , the PE is 0.266 and the CFM is 0.304 .

\section{B. Unraveling some shape properties of $4 D$ modulations}

The 2D graphs obtained from the Cayley-Dickson representation of quaternions are visualization tools that do not allow distinguishing between the constellations obtained by the direct fibration (Figure (4)) or the factorization method (Figure (6)). Possible ambiguities in the representation of QAM partitions comes from the superposition of symbols occurring even in the partition of few vectors. To exemplify, consider for instance the following three vectors $(0,0,1,1),(0,0,0,1),(0,0,1,0)$. After partitioning these vectors, the QAM projection for $\left(x_{1}+i x_{2}\right)$ repeats three times the symbol $(0,0)$ while the projection referring to $\left(x_{3}+i x_{4}\right)$ maps to the three different positions $(1,1),(0,1)$ and $(1,0)$. Although this ambiguity is only a matter of representation we can mitigate it using histograms to count the occurrence of QAM symbols. Examples of this kind of histograms are illustrated respectively in Figures (7) and (8). The histograms in Figures (7) and (8) were calculated using the left side of the quadrature diagrams illustrated, respectively, in the Figure (4) and (6). However, as we know that the base points of this constellations are antipodally symmetric, the right sides would present the same shape. Observing these histograms, one can intuitively conclude that this approach allows the observation of features of $4 \mathrm{D}$ constellations that approximate both probabilistic shaping [33] and geometric shaping [34]. Qualitatively, the probabilistic shape of the 14PolSK-8PSK shows a concentration of symbols near the center of the IQ diagram. This means that symbols with lower norms are generated more frequently than those with higher norms. At the same time, this constellation displays in the IQ diagram a non-uniform distribution of symbols, also revealing characteristics of geometric shaping. The base 2 version reveals that the geometric distribution of symbols was affected by the factorization method and now eight adjacent 


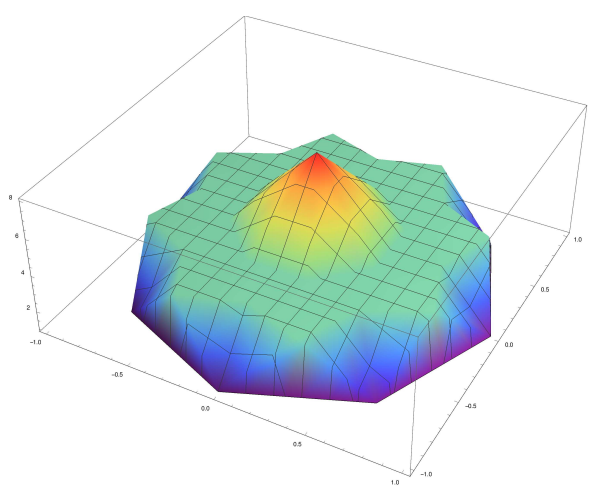

$(a)$

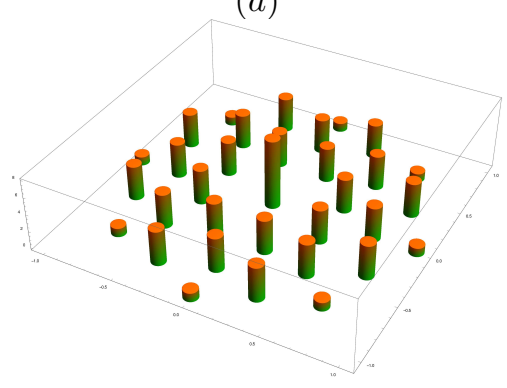

(b)

Fig. 7. Histograms of the 14PolSK-8PSK constellation obtained from the QAM partitions of the unit quaternions.

lobes can be observed around the central lobe. Regarding the shape properties of these constellations, it is important to note that we are dealing here with uncoded modulations, so the finding of this features is of significative relevance.

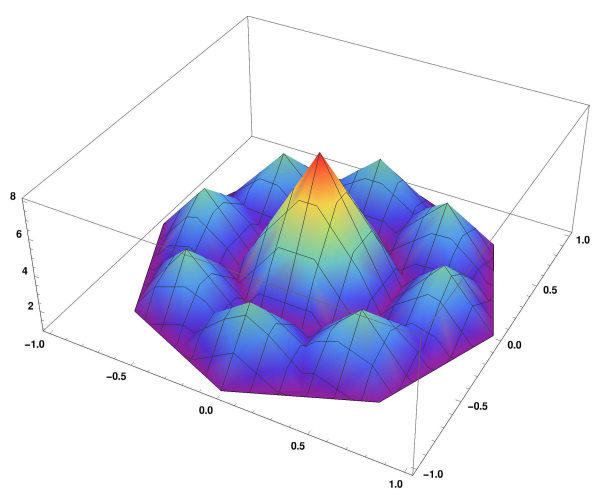

(a)

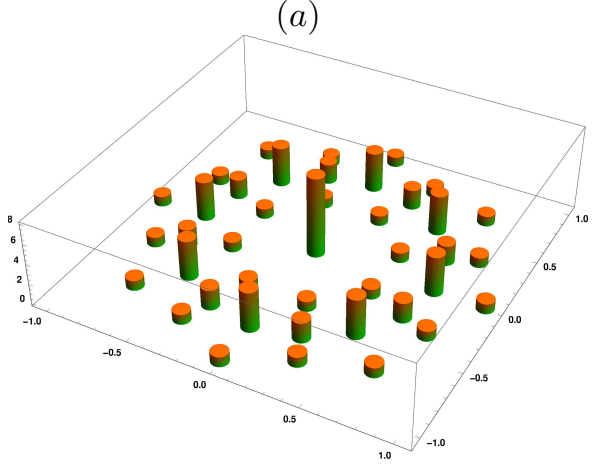

(b)

Fig. 8. Histograms of the (12PolSK-8PSK) + (2PolSK-16PSK) constellation obtained from the QAM partitions of the unit quaternions.

\section{SOME REMARKS ON THE REQUIRED PROPERTIES FOR THE BASE SPACE}

When using the discrete Hopf map one needs to consider which properties of the basis points $S^{2}$ will contributes to the construction of good four-dimensional constellations. In this task, integrated requirements in the optical and electrical domains reveals specific requirements that emerges differently of the usual applications. To exemplify, we present in the following some results from the construction of the 8PolSK-1PSK constellation using two different arrangements of points. In the first example, basis points were defined by vertices of the hexahedron. Vertices of this polytope and the related $4 \mathrm{D}$ vectors generated by the inverse Hopf map are shown in Table (II). One graphical representation of this Hopf map is depicted in the Figure (9). The main idea here is to observe how the metric preservation characteristics of the Hopf map will reflect in its 4D projection [22].

TABLE II

DISCRETE FIBRATION OF THE HEXAHEDRON $\left(\psi_{0}=\frac{\pi}{8}\right)$

\begin{tabular}{|c|c|}
\hline Stokes vectors (basis points) & Unit quaternions (fibration) \\
\hline$(0.577,0.577,0.577)$ & $(0.628,0.628,0.460,0)$ \\
\hline$(-0.577,0.577,0.577)$ & $(0.888,0,-0.325,-0.325)$ \\
\hline$(-0.577,-0.577,0.577)$ & $(0.628,0.628,-0.460,0)$ \\
\hline$(0.577,-0.577,0.577)$ & $(0.888,0,0.325,0.325)$ \\
\hline$(0.577,0.577,-0.577)$ & $(0.325,0.325,0.888,0)$ \\
\hline$(-0.577,0.577,-0.577)$ & $(0.460,0,-0.628,-0.628)$ \\
\hline$(-0.577,-0.577,-0.577)$ & $(0.325,0.325,-0.888,0)$ \\
\hline$(0.577,-0.577,-0.577)$ & $(0.460,0,0.628,0.628)$ \\
\hline
\end{tabular}

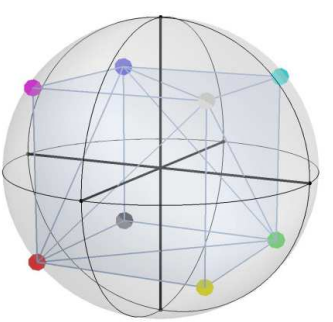

(a)

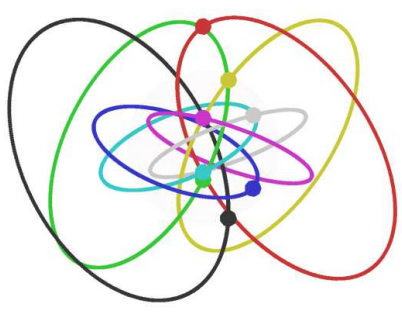

(b)
Fig. 9. Base space with 8 points defined by vertices of the hexahedron embedded in $S^{2}$ and the resulting (b) stereographic projection of the discrete fibration with a single sample.

By isolating single unique values from the coordinates of the unit quaternions represented in the right side of Table (II) we get, in the specific example, the following symmetric vector:

$$
( \pm 0.888, \pm 0.628, \pm 0.460, \pm 0.325)
$$

From the physical perspective, the coordinates of (26) defines the real valuated voltages required to generate our target 
constellation. In addition, these voltages also defines the DAC quantizer that can be considered as a PAM modulation applied in the entries of the PMQ-MZM device. A graphical representation of this quantizer is illustrated in Figure (10).

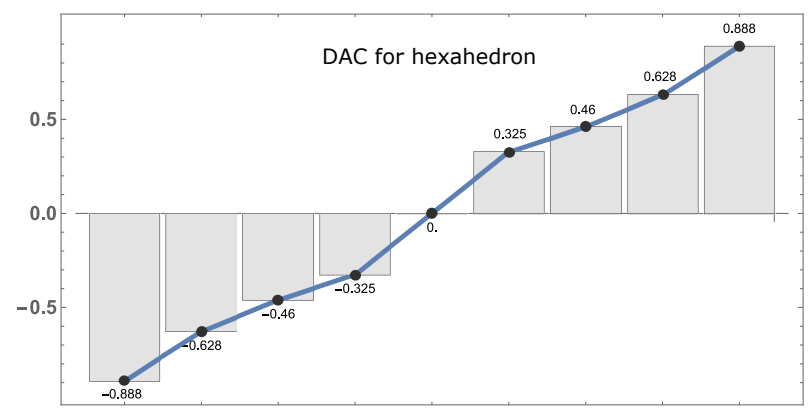

Fig. 10. Quantizer (PAM voltages) of the DAC as an alternative representation of the hexahedron single unique value vector (26).

As a DAC quantizer, vector (26) has 8 levels that emulates a companding function [35].

Doing the same procedure using the square antiprism we get the following Hopf map:

TABLE III

DISCRETE FIBRATION OF THE SQUARE ANTIPRISM $\left(\psi_{0}=\frac{3 \pi}{4}\right)$

\begin{tabular}{|c|c|}
\hline Stokes vectors (basis points) & Unit quaternions (fibration) \\
\hline$(0.577,0.577,0.577)$ & $(-0.8205,0.3399,-0.1759,0.4247)$ \\
\hline$(-0.577,0.577,0.577)$ & $(-0.3399,0.8205,0.4247,-0.1759)$ \\
\hline$(-0.577,-0.577,0.577)$ & $(-0.8205,0.3399,0.1759,-0.4247)$ \\
\hline$(0.577,-0.577,0.577)$ & $(-0.3399,0.8205,-0.4247,0.1759)$ \\
\hline$(0,0.816,-0.577)$ & $(-0.4597,0,0,0.8881)$ \\
\hline$(-0.816,0,-0.577)$ & $(-0.3251,0.3251,0.628,-0.6280)$ \\
\hline$(0,-0.816,-0.577)$ & $(0,0.4597,-0.8881,0)$ \\
\hline$(0.816,0,-0.577)$ & $(-0.3251,0.3251,-0.6280,0.6280)$ \\
\hline
\end{tabular}

The base space and the stereographic projection of the discrete fibration is illustrated in Figure (11)

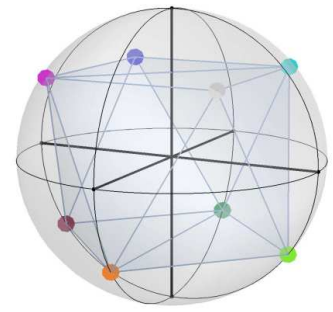

(a)

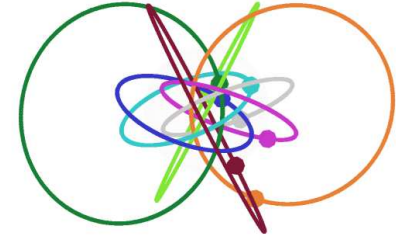

(b)
Fig. 11. (a) Base space with 8 points defined by vertices of the square antiprism embedded in $S^{2}$ and the resulting (b) stereographic projection of the discrete fibration with a single sample.
From the single unique values in the Table (III), the DAC quantizer has 17 levels in a symmetric vector:

$$
\begin{aligned}
& ( \pm 0.888, \pm 0.820, \pm 0.628, \pm 0.460, \ldots \\
& \quad \pm 0.425, \pm 0.340, \pm 0.325, \pm 0.176,0)
\end{aligned}
$$

This vector, in turn, has pictorial representation in Figure (12).

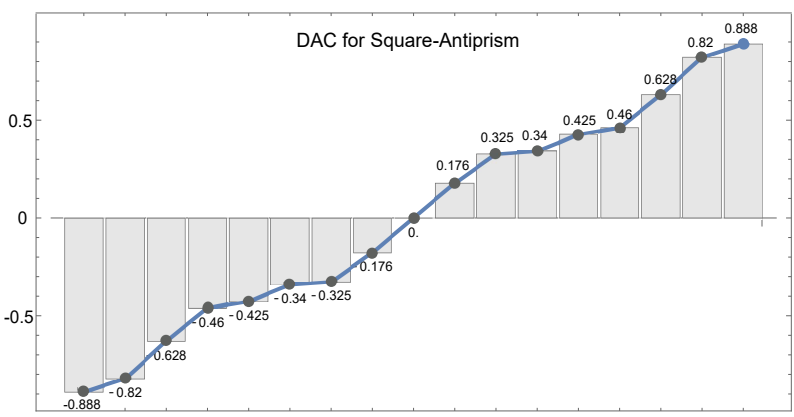

Fig. 12. Quantizer (PAM voltages) of the DAC as an alternative representation of the square antiprism single unique value vector (27).

By comparing the single unique values in vectors (26) and (27), one can intuitively conclude that the choice of the base space poses a tradeoff in the electrical domain independent of the optical properties of constellations. Considering base spaces defined by polytopes, we can also conclude that the cardinality of the DAC can be reduced in cases where the vertex configuration is antipodally symmetric. Finally, it is worth noting that while the example presented here used a simple 8PolSK structure [36], the same relation holds for generic nPolSK-mPSK constellations.

\section{LABELING DISCRETE HOPF MAPS IN NPOLSK-MPSK 4D CONSTELLATIONS}

According to [37], the usage of a single metric for the distance between constellation symbols are not effective in communications over noisy Gaussian channels. So, targeting operations in low SNR regimes, hybrid metrics are interesting solutions in the construction of high-order modulations. In the present work, we consider the use of a hybrid Euclidean-Hamming metric to the labeling of nPolSK-mPSK constellations. The proposed scheme is based on simple covering codes that are largely known in the literature [38], [39]. So, to describe some details of our motivation, a few basic properties of these codes will be presented. For convenience, the graph of the covering code $\{000,111\}$ is illustrated in Figure (13).
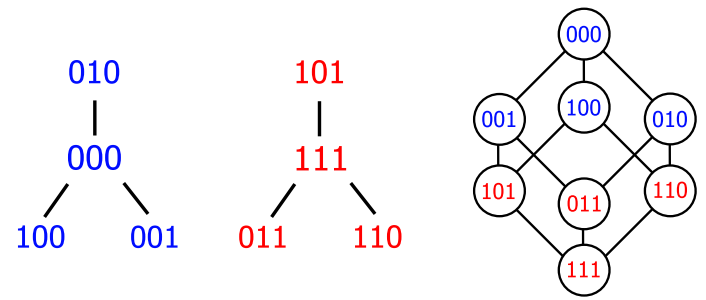

Fig. 13. Example of a perfect binary covering code with order $n=3$ in the vertex labeling of a hypercube- 3 . 
The code $\{000,111\}$ with partitions $a=\{000,001,010,100\}$ and $b=\{111,011,101,110\}$ is a perfect covering code representing all binary combinations of 3 bits. In this code, the Hamming distance between the binary words $\{000\}$ and $\{111\}$ equals 3 , while the distance between symbols in each partition equals 1 . In this sense, if the hypercube-3 graph illustrated in Figure (13) is used to represent vertices of a hexahedron embedded in a unit sphere, then the relation between Euclidean and Hamming distances reveals the hybrid metric described in Table (IV).

TABLE IV

EUCLIDEAN AND HAMMING DISTANCE IN A HYPERCUBE-3 WITH EDGE LENGTH $l$, LABELED WITH A PERFECT COVERING CODE.

\begin{tabular}{|l|c|c|c|}
\hline & Edge & Face diagonal & Main diagonal \\
\hline Euclidean distance & $1 l$ & $\sqrt{2} . l$ & $\sqrt{3} . l$ \\
\hline Hamming distance & 1 & 2 & 3 \\
\hline
\end{tabular}

From Table (IV) we can observe that, for a given Hamming distance $\boldsymbol{d}$, the related Euclidean distance is $\sqrt{\boldsymbol{d}} \cdot \boldsymbol{l}$. Where $l$ is the edge length of the cube. Moreover, when vertices of the referred hexahedron are used as basis points of a 8PolSK-8PSK fibration, the codewords $\{000\}$ and $\{111\}$ may be used to identify antipodal points in the $S^{2}$ base. The whole process can be done using one covering code for the basis points and other similar code for the Hopf fibres. A detailed example of the labeling of a single base point is illustrated in Figure (14). The label (000) was applied to the base point and by means of a de Bruijn sequence [39], each angular coordinate $\psi$ sampled from the Hopf fibre was labeled with a binary codeword of length 3 . In the 8PolSK-8PSK example, the final code is a simple concatenation of codewords (see Table (V) in Appendix (B).

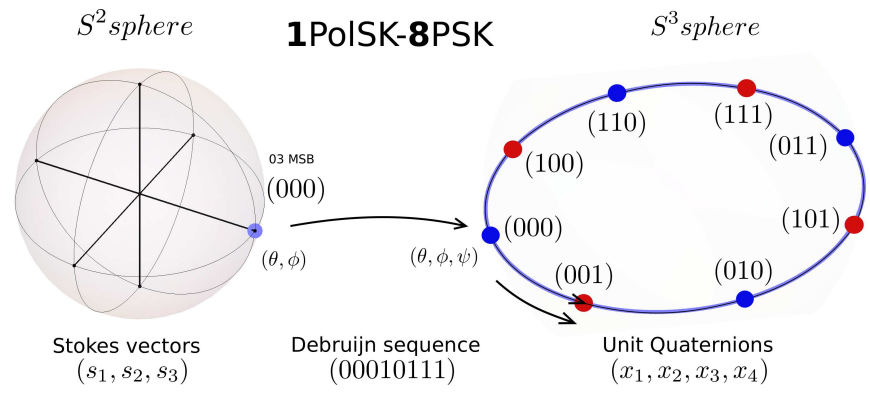

Fig. 14. Labeling of points in sampled Hopf fibres with a covering code of order $n=3$.

In resume, from the concatenated codewords, the most significative (MSB) 3-bit codewords were used to label the basis points and the least significative (LSB) were used to label the angular coordinates (positions) in the Hopf fibre. Since a Hopf fibre is a geometric ring, the analogy with the labeling of PSK modulations is straightforward to consider [40]. It is important to note that the labeling procedure described herein is mainly intended to demonstrate the capabilities of the Hopf fibration. For specialized codes in toroidal structures, one can refer for example [41].
On the Hopf fibration method and its properties applied to the design of 4D modulations the following characteristics and discussions are highlighted:

- Using the inverse Hopf map, the distance between the basis points defines the distance between the great circles where the Hopf fibres reside. So, if a uniform spherical code is used as a basis in $S^{2}$ [42], [43], the fibration of the $4 \mathrm{D}$ modulation will be evenly spaced;

- The symmetry of the basis points defines relevant properties of the DAC quantizer. For example, the square antiprism with 8 vertices is a spherical code with a better minimum distance than the hexahedron. Despite that, the 8PolSK-8PSK constellation designed from the later needs fewer voltage levels to be built. This holds due to the fact that the hexahedron vertices are antipodally symmetric;

- The design of 4D modulations using Hopf fibres is a constructive method that differs from other approaches based solely on permutation of vector coordinates [5], [6], [2]. An advantage of the method presented here is the direct relation between the projected modulations and the physical characteristics of the signal to be transmitted in both the optical domain (Stokes parameters) and in the electrical domain (voltages as coordinates of 4D vectors);

- It is expected that the polarization spectrum of the optical signal [44] will be determined by Stokes vectors defined by vertices of basis polytopes. This polarization spectrum may consequently reflect in channel impairments such as the PDL;

- A polytope composed of subpolytopes in the sphere $S^{3}$ tends to project a polytope composed of subpolytopes in the sphere $S^{2}$, so the embedded vertex property allows the two-way map between nested polytopes [16]. This feature can be easily identified in the 6PolSK-QPSK modulation structure;

- The construction of four-dimensional constellations is strongly influenced by the initial angle $\psi$ used in the inverse Hopf map. This feature can be used to rotate the entire discrete fibration by choosing different angles to start. This is therefore a simple method to optimize the constellation parameters in relation to some desired requirement. The DAC resolution, for example, can be changed by choosing different values of the initial $\psi$. As demonstrated in [8], this procedure is equivalent to the use of a generalized rotation matrix that [11] used, in the specific case, to change the representation coordinates of the 24-Cell;

- The strong dependence on the parametrization gives to the discrete Hopf fibration high flexibility. However, for some applications, it may present some drawbacks. Concerning this property, it is expected that the methods presented here can find better use in the construction of fiber optic constellations aiming for concurrent integrated requirements in both optical and electrical domains; 


\section{CONCLUSIONS}

Some properties of the Hopf fibration and the embedded vertex concept were demonstrated in the design of four-dimensional modulations restricted to the scope of classical coherent optical communications. In the constructive methods presented, the fibration of the sphere $S^{3}$ was realized using the parametrization of basis points defined by Stokes vectors in the Poincaré sphere $\left(S^{2}\right)$. The Stokes vectors, in turn, were associated with simple structured spherical codes defined by vertices of polytopes. Using the 14PolSK-8PSK modulation as an example, it was found that the explicit connection between the distance of the basis points $S^{2}$ and the distance between the Hopf fibres provides a metric preserving method for the design of four-dimensional constellations. One simple but powerful extension of the standard Hopf fibration method have demonstrated that the factorization in the number of samples in each Hopf fibre leads to the construction of base 2 constellations even in cases where the number of basis points are not base 2 . The related factorization procedure was used in the construction of a 128 symbol constellation. Using the inverse Hopf map, it was demonstrated how the geometry of the base space $S^{2}$ can impact the construction of four-dimensional constellations. The main result being the fact that a base space with antipodally symmetric points allows the construction of modulations with less stringent DAC requirements. A labeling scheme based on simple covering codes was described and demonstrated. It was clear from the expositions across the text that the discrete fibration and the labeling procedure obeys the same geometric nature. The labeling of the 8PolSK-8PSK was realized using a concatenated (nested) code. Finally, based on the results demonstrated, it was concluded that the constructive methods presented here are of practical interest in classical coherent optical communications.

\section{APPENDIX A}

\section{THE CONNECTION BETWEEN SU(2), SO(3) AND QUATERNIONS}

In the sequence, the Euler parameterization of an arbitrary matrix of an $\mathrm{SU}(2)$ transformation is succinctly presented considering the standard Pauli matrices:

$$
\sigma_{1}=\left[\begin{array}{cc}
0 & 1 \\
1 & 0
\end{array}\right] ; \quad \sigma_{2}=\left[\begin{array}{cc}
0 & -i \\
i & 0
\end{array}\right] ; \quad \sigma_{3}=\left[\begin{array}{cc}
1 & 0 \\
0 & -1
\end{array}\right]
$$

which satisfy the relation $\sigma_{i} \sigma_{j}=\delta_{i j}+i e_{i j k}$, with $\delta_{i j}$ and $i e_{i j k}$ being the Dirac and Kronecker delta symbols respectively.

The starting point, in our context, is the exponential map:

$$
\begin{aligned}
U_{2} & =\exp \left(\frac{\mathbf{i} \sigma_{3} \phi}{2}\right) \exp \left(\frac{\mathbf{i} \sigma_{2} \theta}{2}\right) \exp \left(\frac{\mathbf{i} \sigma_{3} \psi}{2}\right) \\
& =U(\phi, \theta, \psi) \\
& =U_{z}(\phi) U_{y}(\theta) U_{z}(\psi)
\end{aligned}
$$

where the notation $U_{z}(\phi) U_{y}(\theta) U_{z}(\psi)$ represents the extrinsic rotations 3-2-3 (z-y-z) [45], [46], [47].

The exponential map in Equation (29) relates $\mathrm{SU}(2)$ to $\mathrm{SO}(3)$ through the explicit product of rotation matrices in $\mathbb{R}^{3}$.

$$
\begin{aligned}
& U(\phi, \theta, \psi)=U_{\left(R_{\phi} R_{\theta} R_{\psi}\right)} \\
& R_{\phi}=\left(\begin{array}{ccc}
\cos (\phi) & -\sin (\phi) & 0 \\
\sin (\phi) & \cos (\phi) & 0 \\
0 & 0 & 1
\end{array}\right) \\
& R_{\theta}=\left(\begin{array}{ccc}
\cos (\theta) & 0 & \sin (\theta) \\
0 & 1 & 0 \\
-\sin (\theta) & 0 & \cos (\theta)
\end{array}\right) \\
& R_{\psi}=\left(\begin{array}{ccc}
\cos (\psi) & -\sin (\psi) & 0 \\
\sin (\psi) & \cos (\psi) & 0 \\
0 & 0 & 1
\end{array}\right)
\end{aligned}
$$

Alternatively, Equation (30) can be written in the form:

$$
\begin{aligned}
R_{\phi} & =\left(\begin{array}{cc}
e^{i\left(\frac{\phi}{2}\right)} & 0 \\
0 & e^{-i\left(\frac{\phi}{2}\right)}
\end{array}\right) \\
R_{\theta} & =\left(\begin{array}{cc}
\cos \left(\frac{\theta}{2}\right) & \sin \left(\frac{\theta}{2}\right) \\
-\sin \left(\frac{\theta}{2}\right) & \cos \left(\frac{\theta}{2}\right)
\end{array}\right) \\
R_{\psi} & =\left(\begin{array}{cc}
e^{i\left(\frac{\psi}{2}\right)} & 0 \\
0 & e^{-i\left(\frac{\psi}{2}\right)}
\end{array}\right) \\
U(\phi, \theta, \psi) & = \pm\left(\begin{array}{cc}
\cos \left(\frac{\theta}{2}\right) e^{i\left(\frac{\phi+\psi}{2}\right)} & \sin \left(\frac{\theta}{2}\right) e^{i\left(\frac{\phi-\psi}{2}\right)} \\
-\sin \left(\frac{\theta}{2}\right) e^{-i\left(\frac{\phi-\psi}{2}\right)} & \cos \left(\frac{\theta}{2}\right) e^{-i\left(\frac{\phi+\psi}{2}\right)}
\end{array}\right)
\end{aligned}
$$

If the extrinsic rotations in Equations (29), (30) and (31) are chosen to be $U_{z}(\phi) U_{x}(\theta) U_{z}(\psi)$ 3-1-3 (z,x,z), the rotation provided by the exponential map will be body centered and, in this case, Equation (31) will be in accordance with [21]:

$$
U(\phi, \theta, \psi)= \pm\left(\begin{array}{cc}
\cos \left(\frac{\theta}{2}\right) e^{i\left(\frac{\phi+\psi}{2}\right)} & i \sin \left(\frac{\theta}{2}\right) e^{i\left(\frac{\phi-\psi}{2}\right)} \\
-i \sin \left(\frac{\theta}{2}\right) e^{-i\left(\frac{\phi-\psi}{2}\right)} & \cos \left(\frac{\theta}{2}\right) e^{-i\left(\frac{\phi+\psi}{2}\right)}
\end{array}\right)
$$

Equation (32) provides the Cayley-Klein parameters in terms of the Euler angles. So, is interesting to note that, depending on the convention adopted, the inverse Hopf fibration $S^{2} \rightarrow S^{3}$ can be viewed as Euler angle representations for the four real Euler parameters: 


$$
\begin{aligned}
& x_{1}=\cos \left(\frac{\theta}{2}\right) \cos \left(\frac{\psi+\phi}{2}\right) ; \\
& x_{2}=\cos \left(\frac{\theta}{2}\right) \sin \left(\frac{\psi+\phi}{2}\right) ; \\
& x_{3}=\sin \left(\frac{\theta}{2}\right) \cos \left(\frac{\psi-\phi}{2}\right) ; \\
& x_{4}=\sin \left(\frac{\theta}{2}\right) \sin \left(\frac{\psi-\phi}{2}\right) ;
\end{aligned}
$$

Finally, is straightforward to conceive that both Equations (31) and (32) turns into the general SU(2) representation for complex variables $z_{1}$ and $z_{2}$, where $z_{1}=x_{1}+i x_{2}$ and $z_{2}=x_{3}+i x_{4}$. With $\left(x_{1}, x_{2}, x_{3}, x_{4}\right)$ being quaternion coordinates:

$$
U_{2}=\left(\begin{array}{cc}
z_{1} & z_{2} \\
-\bar{z}_{2} & \bar{z}_{1}
\end{array}\right)
$$

where $z_{1}, z_{2} \in \mathbb{C}$ and $\left|z_{1}\right|^{2}+\left|z_{2}\right|^{2}=1$

\section{APPENDIX B}

\section{SOME TIPS ABOUT THE 8POLSK-8PSK CONSTELLATION}

\section{A. Relations between basis points and the Cayley-Dickson projection - hexahedron vs square antiprism example}

Figures (15a) and (15b) illustrates how the distribution of points in the base space $S^{2}$ changes the Cayley-Dickson projection of the 8PolSK-8PSK.

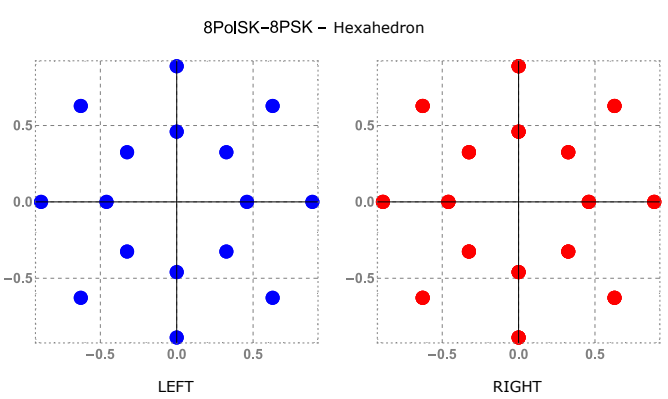

$(a)$

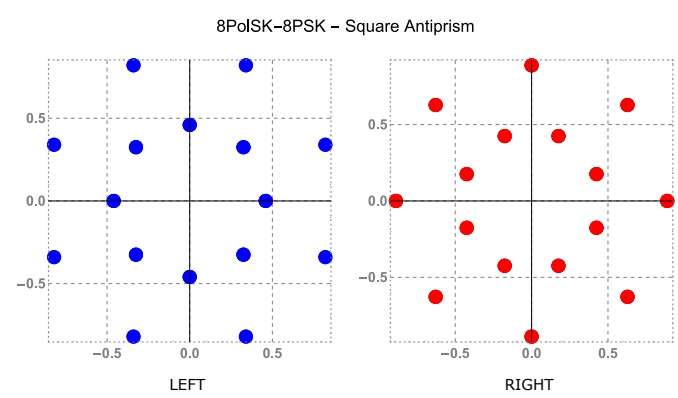

(b)

Fig. 15. (a) Symmetric partitions in two quadrature planes obtained from 64 quaternions using the hexahedron as base space. (b) Non-symmetric (rotated) partitions in two quadrature planes obtained from 64 quaternions using the square antiprism as base space.
In this example, the vertex structures of the hexahedron and the square antiprism were alternately used in the definition of points in the base space. For simplicity, we consider both polytopes as the composition of two parallel squares embedded in $S^{2}$. In this way, considering a common axis perpendicular to these squares, the antiprism have a relative rotation of $\pi / 4$ over the hexahedron. After the generation of each fibration, the resulting effect in the geometry of the four-dimensional 8PolSK-8PSK constellation could be observed in the Cayley-Dickson projection as a rotation in the quadrature plane. (left and right sides of Figure (15b)). The rigid angular displacement between the left and right projections is in turn $0.5 \times(2 \pi / 8)=\pi / 8$, that is by half the value of the relative rotation between the considered parallel squares.

\section{B. Binary labels from concatenated covering codes}

The simple covering code shown in Table $(\mathrm{V})$ originated from two codes. First, a Gray code along the Hamiltonian path illustrated in the graph of Figure (16) was used to label points in the base. The second code, obtained from a de Bruijn sequence of length 3 over a binary alphabet was used to label angular positions sampled in the Hopf fibres [39]. The resulting labels with length 6 are thus concatenated codewords. Since the two codes were applied over rings, the final code can reside in a toric structure with 64 nodes (Figure 17).

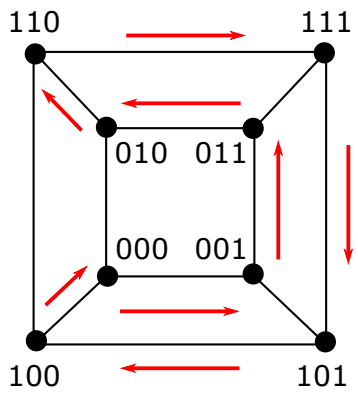

Fig. 16. Graph of the Hamiltonian path over the hypercube-3 (hexahedron). The labels along the path comes from a binary Gray code.

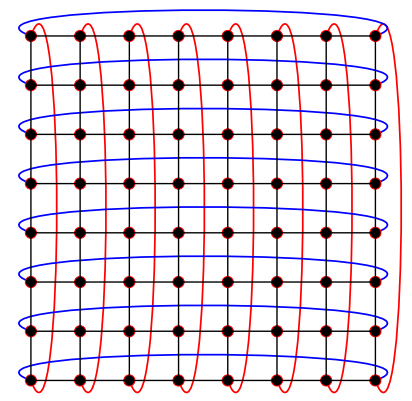

Fig. 17. Toric structure with 64 nodes that can be associated with the resulting code shown in Table (V). Rows are related to the angular positions sampled in the Hopf fibres while columns are related to the Hamiltonian path over the hypercube-3 (hexahedron) 
TABLE V

BINARY LABELING OF THE 8POLSK-8PSK CONSTELLATION USING CONCATENATED CODEWORDS FROM COVERING CODES.

\begin{tabular}{|c|c|c|c|c|c|c|c|c|}
\hline \multicolumn{8}{|c|}{ Basis points $($ MSB $) \leftarrow$} & \\
\hline 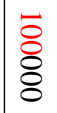 & 표 & $\begin{array}{l}0 \\
8 \\
8 \\
8\end{array}$ & 응 & 局 & $\mid \begin{array}{l}\overrightarrow{0} \\
\overline{8} \\
8\end{array}$ & $\begin{array}{l}8 \\
8 \\
8 \\
8\end{array}$ & $\begin{array}{l}8 \\
8 \\
8 \\
8\end{array}$ & \\
\hline ఫे & 훙 & ○ & 응 & 居 & $\begin{array}{l}\vec{o} \\
\bar{o} \\
\varrho\end{array}$ & $\begin{array}{l}8 \\
8 \\
8\end{array}$ & \& & \\
\hline $\begin{array}{l}\text { ठ্ } \\
\vdots \\
0\end{array}$ & $\begin{array}{l}\vec{\Xi} \\
\stackrel{0}{0} \\
\end{array}$ & $\left(\begin{array}{l}0 \\
\vdots \\
0 \\
0\end{array}\right.$ & 号 & 咅 & $\begin{array}{l}0 \\
0 \\
0 \\
0\end{array}$ & $\begin{array}{l}8 \\
0 \\
0 \\
0\end{array}$ & $\begin{array}{l}8 \\
\vdots \\
0 \\
0\end{array}$ & $\begin{array}{l}\downarrow \\
\mathscr{\infty}\end{array}$ \\
\hline$\frac{\overline{8}}{8}$ & $\begin{array}{l}\overrightarrow{0} \\
0 \\
0\end{array}$ & $\begin{array}{l}0 \\
0 \\
0 \\
0\end{array}$ & 异 & $\begin{array}{l}\Xi \\
\Xi \\
\Xi\end{array}$ & $\left(\begin{array}{l}\overrightarrow{0} \\
\stackrel{0}{\ominus}\end{array}\right.$ & $\begin{array}{l}8 \\
\varrho \\
\varrho \\
\varrho\end{array}$ & \begin{tabular}{l}
8 \\
8 \\
\hdashline
\end{tabular} & 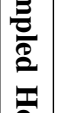 \\
\hline ‡ & $\bar{\Xi}$ & $\stackrel{\circ}{8}$ & 응 & 可 & $\left(\begin{array}{c}0 \\
0 \\
\varrho\end{array}\right.$ & $\stackrel{8}{\circ}$ & 㐫 & $\vec{z}$ \\
\hline $\begin{array}{l}\text { ळ } \\
\Xi\end{array}$ & 哥 & $\stackrel{ }{O}$ & 兰 & $\Xi$ & $\stackrel{\overrightarrow{0}}{\Xi}$ & $\stackrel{8}{\stackrel{8}{\Xi}}$ & 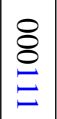 & $\underset{50}{50}$ \\
\hline$\frac{\overline{8}}{\sigma}$ & 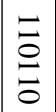 & $\begin{array}{l}0 \\
0 \\
O \\
0\end{array}$ & 证 & ت & 官 & $\begin{array}{l}\stackrel{8}{\circ} \\
\Xi \\
\end{array}$ & $\begin{array}{l}8 \\
\varnothing \\
ٍ \\
0\end{array}$ & \\
\hline $\begin{array}{l}\overline{8} \\
\overline{8}\end{array}$ & 豙 & $\begin{array}{l}0 \\
0 \\
8 \\
8\end{array}$ & 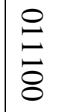 & $\Xi$ & $\left(\begin{array}{l}\sigma \\
\overline{8}\end{array}\right.$ & $\begin{array}{l}8 \\
\stackrel{8}{8} \\
8\end{array}$ & $\frac{8}{8}$ & \\
\hline
\end{tabular}

C. Numerical example of a 8PolSK-8PSK constellation

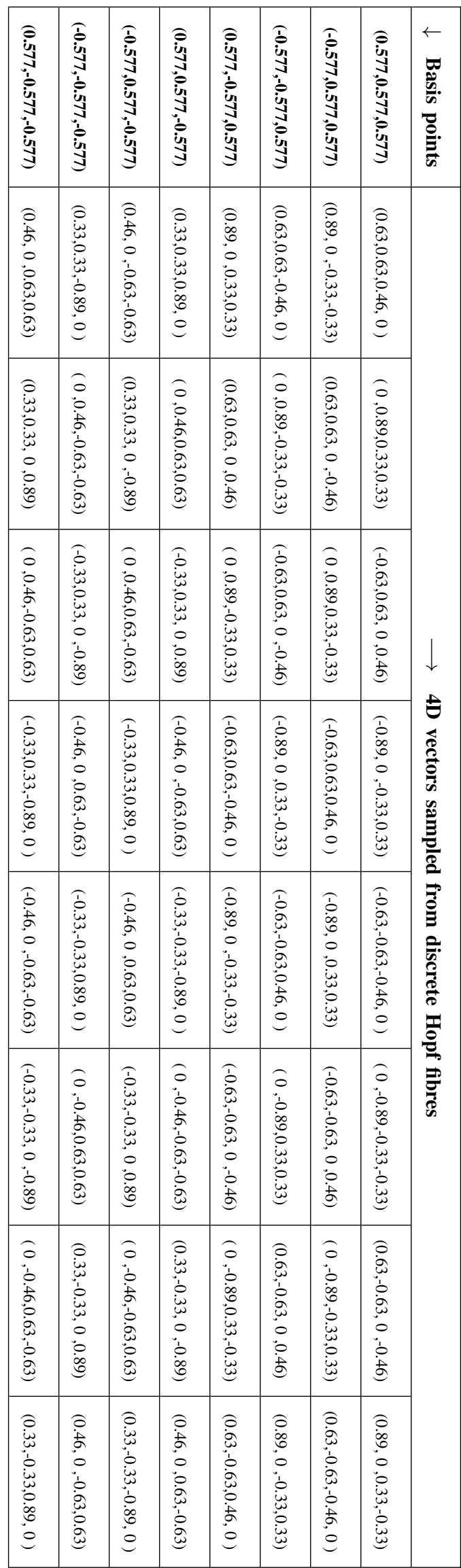




\section{ACKNOWLEDGMENT}

The authors would like to thank the Brazilian funding agency CAPES, the Scientific and Industrial Metrology Board (Dimci) of INMETRO and the Center for Telecommunications Studies (CETUC/PUC-Rio).

\section{REFERENCES}

[1] P. Johannisson, M. Sjodin, T. A. Eriksson, and M. Karlsson, "Four-dimensional modulation formats for long-haul transmission," in Conference on Optical Fiber Communication, Technical Digest Series, 2014, doi: https://doi.org/10.1364/OFC.2014.M2C.4.

[2] S. Ishimura and K. Kikuchi, "Multi-Dimensional Permutation-Modulation Format for Coherent Optical Communications," Opt. Express, vol. 23, no. 12, pp. 15587-15597, jun 2015, doi: https://doi.org/10.1364/OE.23.015587.

[3] P. J. Winzer, "Beyond 100G Ethernet," IEEE Communications Magazine, vol. 48, no. 7, pp. 26-30, jul 2010, doi: https://doi.org/10.1109/MCOM. 2010.5496875

[4] T. Optical and I. Forum, "Technology Options for 400G Implementation," Forum, no. July, pp. 1-46, 2015.

[5] G. Welti and J. Lee, "Digital transmission with coherent four-dimensional modulation," IEEE Transactions on Information Theory, vol. 20, no. 4, pp. 497-502, jul 1974, doi: https://doi.org/10.1109/TIT.1974.1055247.

[6] L. Zetterberg and H. Brandstrom, "Codes for Combined Phase and Amplitude Modulated Signals in a Four-Dimensional Space," IEEE Transactions on Communications, vol. 25, no. 9, pp. 943-950, sep 1977 doi: https://doi.org/10.1109/TCOM.1977.1093927.

[7] M. Visintin, E. Biglieri, and V. Castellani, "Four-dimensional signaling for bandlimited channels," IEEE Transactions on Communications, vol. 42, no. 234, pp. 403-409, feb 1994, doi: https://doi.org/10.1109/ TCOMM.1994.577067.

[8] F. A. Rodrigues, G. P. Temporão, and J. P. von der Weid, "Discrete Hopf Fibrations in the Signal Space of Four-Dimensional Modulations," in Advanced Photonics 2017 (IPR, NOMA, Sensors, Networks, SPPCom, $P S)$. Optical Society of America, 2017, p. SpTu2F.3, doi: https://doi. org/10.1364/SPPCOM.2017.SpTu2F.3.

[9] F. A. Rodrigues, G. Temporão, and J. P. von der Weid, "Discrete Hopf Fibration in the Design of Four-Dimensional Modulations," in 2017 SBMO/IEEE MTT-S International Microwave and Optoelectronics Conference (IMOC), Aug 2017, pp. 1-5, doi: https://doi.org/10.1109/ IMOC.2017.8121087.

[10] F. A. Rodrigues, G. P. Temporão, and J. P. von der Weid, "Fibrações Hopf e Modulações 4D (in Portuguese)," in XXXV Simpósio Brasileiro de Telecomunicações e Processamento de Sinais - SBRT 2017, Sept 2017, pp. 663-667. [Online]. Available: http://www.sbrt.org.br/sbrt2017/anais/1570361847.pdf

[11] E. Agrell and M. Karlsson, "On the symbol error probability of regular polytopes," IEEE Transactions on Information Theory, vol. 57, no. 6, pp 3411-3415, June 2011, doi: https://doi.org/10.1109/TIT.2011.2134290.

[12] D. W. Lyons, "An Elementary Introduction to the Hopf Fibration," Mathematics Magazine, vol. 76, no. 2, pp. 87-98, 2003, doi: https: //doi.org/10.2307/3219300.

[13] K. Durstberger, "Geometry of entanglement and decoherence in quantum systems," Ph.D. dissertation, Universität Wien, 2005. [Online]. Available: http://www.phryger.at/kadu/downloads/Dissertation.pdf

[14] J. V. Waite, "The hopf fibration and encoding torus knots in light fields," Master's thesis, University of Nevada, Las Vegas, 2016. [Online]. Available: http://digitalscholarship.unlv.edu/thesesdissertations/2756/

[15] C. Bartocci, L. Boi, and C. Sinigaglia, New Trends in Geometry: Their Role in the Natural and Life Sciences. Imperial College Press, 2011, ch. The Geometry of Dense Packing and Biological Structures.

[16] J. Adams, P. Zvengrowski, and P. Laird, "Vertex Embeddings of Regular Polytopes," Expositiones Mathematicae, vol. 21, no. 4, pp. 339-353, 2003, doi: https://doi.org/10.1016/S0723-0869(03)80037-3.

[17] M. R. Dennis and M. A. Alonso, "Swings and Roundabouts: Optical Poincaré Spheres for Polarization and Gaussian Beams," Philosophical Transactions of the Royal Society A: Math., Phys. and Eng. Sciences, vol. 375, no. 2087, jan 2017, doi: https://doi.org/10.1098/rsta.2015.0441.

[18] H. K. Urbantke, "The Hopf fibration - seven times in physics," Journal of Geometry and Physics, vol. 46, no. 2, pp. 125-150, 2003.

[19] B. Jurčo, "Polarization of Light and Hopf Fibration," Czechoslovak Journal of Physics, vol. 37, no. 9, pp. 1035-1038, 1987, doi: https: //doi.org/10.1007/BF01597447.
[20] C. Torezzan, S. I. R. Costa, and V. A. Vaishampayan, "Constructive Spherical Codes on Layers of Flat Tori," IEEE Transactions on Information Theory, vol. 59, no. 10, pp. 6655-6663, Oct 2013, doi: http://doi.org/10.1109/TIT.2013.2272931.

[21] E. Wolf, Appendix A: Lie Groups in Polarization Optics, ser. Progress in Optics. Elsevier Science, 2006, no. vol. 49, pp. 368-371.

[22] G. Rote, Congruence Testing of Point Sets in Three and Four Dimensions. Cham: Springer International Publishing, 2016, pp. 50-59, doi: https://doi.org/10.1007/978-3-319-32859-1_4.

[23] J. H. Conway and N. J. A. Sloane, Sphere Packings, Lattices and Groups. New York, NY: Springer New York, 1999, ch. Coverings, Lattices and Quantizers, pp. 31-62, doi: https://doi.org/10.1007/978-1-4757-6568-7_ 2.

[24] M. Seimetz, "Transmitter Design," in High-Order Modulation for Optical Fiber Transmission, ser. Springer Series in Optical Sciences. Springer Berlin Heidelberg, 2009.

[25] A. Chen and E. Murphy, Broadband Optical Modulators: Science, Tech., and Applications. CRC Press, 2011.

[26] OIF, "Implementation Agreement for Integrated Polarization Multiplexed Quadrature Modulated Transmitters v01.1," Optical Internetworking Forum, Tech. Rep., 2013. [Online]. Available: http://www.oiforum.com/public/impagreements.html

[27] T. A. Ell, N. L. Bihan, and S. J. Sangwine, Quaternion Fourier Transforms for Signal and Image Processing, ser. FOCUS Series. Wiley, 2014, ch. Quaternion Algebra, pp. 1-19.

[28] J. Conway, H. Burgiel, and C. Goodman-Strauss, The Symmetries of Things, ser. Ak Peters Series. Taylor \& Francis, 2008, ch. Naming the Archimedean and Catalan polyhedra and tilings, p. 284.

[29] M. Chagnon, M. Osman, Q. Zhuge, X. Xu, and D. V. Plant, "Analysis and experimental demonstration of novel 8PolSK-QPSK modulation at 5 bits/symbol for passive mitigation of nonlinear impairments," Opt. Express, vol. 21, no. 25, pp. 30204-30220, Dec 2013, doi: https://doi. org/10.1364/OE.21.030204.

[30] S. J. Sangwine and N. L. Bihan, "Quaternion polar representation with a complex modulus and complex argument inspired by the Cayley-Dickson form," Advances in Applied Clifford Algebras, vol. 20, no. 1, pp. 111-120, 2010, doi: https://doi.org/10.1007/ s00006-008-0128-1.

[31] X. Zhou and C. Xie, Enabling Technologies for High Spectral-efficiency Coherent Optical Communication Networks, ser. Wiley Series in Microwave and Optical Engineering. Wiley, 2016, ch. Multidimensional Optimized Optical Modulation Formats, doi: https://doi.org/10.1002/ 9781119078289.

[32] M. Karlsson and E. Agrell, "Which is the most power-efficient modulation format in optical links?" Optics Express, vol. 17, no. 13, pp. 10 814-10 819, 2009, doi: https://doi.org/10.1364/OE.17.010814.

[33] T. Fehenberger, "Information rates of probabilistically shaped coded modulation for a multi-span fiber-optic communication system with 64QAM," Optics Communications, vol. 409, pp. 2-6, 2018, doi: https: //doi.org/10.1016/j.optcom.2017.07.039.

[34] S. Zhang and F. Yaman, "Constellation design with geometric and probabilistic shaping," Optics Communications, vol. 409, pp. 7-12, 2018, doi: https://doi.org/10.1016/j.optcom.2017.08.063.

[35] B. Smith, "Instantaneous Companding of Quantized Signals," Bell System Tech. Journal, vol. 36, no. 3, pp. 653-709, 1957, doi: https: //doi.org/10.1002/j.1538-7305.1957.tb03858.x.

[36] S. Benedetto and P. Poggiolini, "Polarization Shift Keying: An Efficient Coherent Optical Modulation," in SBT/IEEE International Symposium on Telecommunications, Sep 1990, pp. 14-20, doi: https://doi.org/10. 1109/ITS.1990.175565

[37] X. Zhang, Y. Zhao, and L. Zou, "Optimum TCM Codes Design for Gaussian Channels by Considering Both Euclidean and Hamming Distances," in Communications, 2009. ICC '09. IEEE International Conference on, jun 2009, pp. 1-5, doi: https://doi.org/10.1109/ICC.2009. 5199298.

[38] P. R. J. Östergård and W. D. Weakley, "Classification of Binary Covering Codes," Journal of Combinatorial Designs, vol. 8, no. 6, pp. 391-401, 2000, doi: https://doi.org/10.1002/1520-6610(2000)8:6<391:: AID-JCD1) 3.0.CO;2-R

[39] C. Fan and C. J. N., "De Bruijn cycles for covering codes," Random Structures \& Algorithms, vol. 25, no. 4, pp. 421-431, 2004, doi: https: //doi.org/10.1002/rsa.20033.

[40] E. Agrell, J. Lassing, E. G. Strom, and T. Ottosson, "The binary reflected Gray code is optimal for M-PSK," in International Symposium onInformation Theory, 2004. ISIT 2004. Proceedings., jun 2004, pp. 164-, doi: https://doi.org/10.1109/ISIT.2004.1365203. 
[41] X. Yang, J. Li, H. Du, and J. Han, "A node encoding of Torus topology and its improved routing algorithm," in Journal of Communications, 2008, doi: $10.4304 / \mathrm{jcm} .3 .6 .3-10$.

[42] R. Hardin, N. J. Sloane, and W. D. Smith, "A library of putatively optimal coverings of the sphere with n equal caps," 1987.

[43] R. Hardin, N. Sloane, and W. Smith, "Tables of spherical codes with icosahedral symmetry," 2012. [Online]. Available: http://neilsloane. com/icosahedral.codes/

[44] J. Flamant, P. Chainais, and N. L. Bihan, "Polarization spectrogram of bivariate signals," in 2017 IEEE International Conference on Acoustics, Speech and Signal Processing (ICASSP), mar 2017, pp. 3989-3993, doi: https://doi.org/10.1109/ICASSP.2017.7952905.

[45] H. Goldstein and C. Poole, The Cayley-Klein Parameters and Related Quantities, ser. Addison-Wesley series in physics. Addison-Wesley Publishing Company, 1980.

[46] K. Shoemake, "Animating rotation with quaternion curves," $A C M$ SIGGRAPH Computer Graphics, 1985, doi: https://doi.org/10.1145/ 325165.325242.

[47] D. J. Siminovitch, "Rotations in NMR: Part I. Euler-Rodrigues parameters and quaternions," Concepts in Magnetic Resonance, 1997, doi: https:/doi.org/10.1002/(SICI)1099-0534(1997)9:3〈149:: AID-CMR3 3.0.CO;2-Z.

Fernando Alves Rodrigues was born in Brazil in 1968. He received the B.Sc in Electrical Engineering degree from the State University of Rio de Janeiro (UERJ) in 2003. From 2004 to 2008 he worked in regulatory activities at the National Telecommunications Agency (ANATEL). In 2010 he received a post-graduate specialization in Automation and Instrumentation from the SENAI Institute for Superior Education and in 2012 he received the M.Sc. degree in Electrical Engineering from the Pontifical Catholic University of Rio de Janeiro (PUC-Rio), where he is pursuing the Ph.D. Since 2008, he is working as Technologist Researcher at the Scientific and Industrial Metrology Board (DIMCI) of the National Institute of Metrology, Quality and Technology (Inmetro). His current research interests are metrology, fiber optic communications, high order modulations, rate-adaptive and energy efficient communications.

Guilherme P. Temporão was born in Rio de Janeiro, Brazil, 1980. He received his $\mathrm{PhD}$ degree in Electrical Engineering from the Pontifical Catholic University of Rio de Janeiro (PUC-Rio) in 2007. In 2004-2006 he worked on nonlinear optics and single-photon detectors on the Group of Applied Physics in the University of Geneva, Switzerland. He is currently an associate professor at PUC-Rio and a member of the Optoelectronics and Instrumentation Group since 2008. His current areas of interest are polarization-encoded quantum communication and optical fiber metrology and reflectometry techniques.

Jean Pierre von der Weid Professor Jean Pierre von der Weid obtained his $\mathrm{PhD}$ at the Physics Department of the Pontifical Catholic University of Rio de Janeiro (PUC-Rio) in 1976. Head of the Optoelectronics and instrumentation Group at the same university, he published more than 250 papers in refereed journals and conference proceedings, has 15 patents and patent applications, as well as 14 technologies transferred to industry in the fields of optical fibers, and instrumentation. Senior member of the OSA, IEEE and the Brazilian Academy of Sciences he was awarded as Commander of the Brazilian National Order of Scientific Merit. His current areas of interest are Optical and Quantum Communications, Fiber Sensors and Optical Metrology. 